\title{
Optical alignment techniques for line-imaging velocity interferometry and line-imaging self-emission of targets at the National Ignition Facility (NIF)
}

\author{
Robert M. Malone $^{\mathrm{a}}$, John R. Celeste ${ }^{\mathrm{b}}$, Peter M. Celliers ${ }^{\mathrm{b}}$, Brent C. Frogget ${ }^{\mathrm{a}}$, Robert L. Guyton ${ }^{\mathrm{c}}$, \\ Morris I. Kaufman ${ }^{\mathrm{a}}$, Tony L. Lee ${ }^{\mathrm{b}}$, Brian J. MacGowan ${ }^{\mathrm{b}}$, Edmund W. Ng ${ }^{\mathrm{b}}$, Imants P. Reinbachs ${ }^{\mathrm{c}}$, \\ Ronald B. Robinson $^{\mathrm{b}}$, Thomas W. Tunnell ${ }^{\mathrm{a}}$, Phillip W. Watts ${ }^{\mathrm{c}}$ \\ ${ }^{a}$ National Security Technologies, LLC, P.O. Box 809, 182 East Gate Dr., Los Alamos, NM 87544; \\ ${ }^{\mathrm{b}}$ Lawrence Livermore National Laboratory, P.O. Box 808, Livermore, CA 94551; \\ ${ }^{\mathrm{c}}$ National Security Technologies, LLC, 161 S. Vasco Rd., Livermore, CA 94551
}

\begin{abstract}
The National Ignition Facility (NIF) requires optical diagnostics for measuring shock velocities in shock physics experiments. The nature of the NIF facility requires the alignment of complex three-dimensional optical systems of very long distances. Access to the alignment mechanisms can be limited, and any alignment system must be operatorfriendly. The Velocity Interferometer System for Any Reflector (VISAR) measures shock velocities, shock breakout times, and emission of 1- to 5-mm targets at a location remote to the NIF target chamber. Three optical systems using the same vacuum chamber port each have a total track of $21 \mathrm{~m}$. All optical lenses are on kinematic mounts or sliding rails, enabling pointing accuracy of the optical axis to be checked. Counter-propagating laser beams (orange and red) align these diagnostics to a listing of tolerances. Movable aperture cards, placed before and after lens groups, show the spread of alignment spots created by the orange and red alignment lasers. Optical elements include 1-in. to 15-in.
\end{abstract}

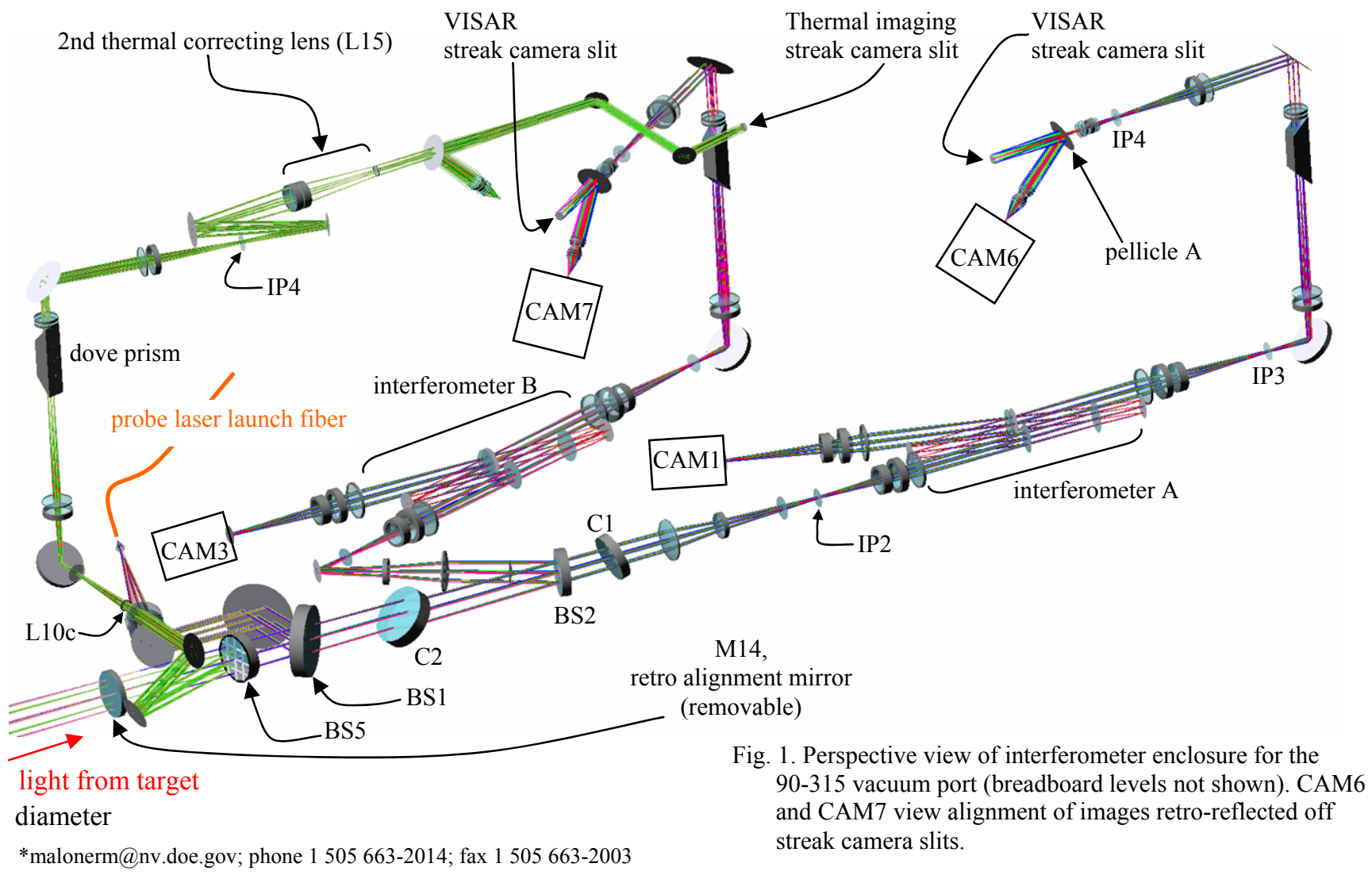


mirrors, lenses with up to 10.5-in. diameters, beamsplitters, etalons, dove prisms, filters, and pellicles. Alignment of more than 75 optical elements must be verified before each target shot. Archived images from eight alignment cameras prove proper alignment before each shot.

Keywords: VISAR, optical relay, optical alignment, optical tolerancing, interferometer, National Ignition Facility (NIF), streak camera

\section{INTRODUCTION}

Figure 1 shows a subset of an optical imaging system that includes beamsplitters to separate light (single wavelength) into two different interferometers, each having its own recording camera, and also to separate other emission light (at a band of wavelengths different from that of the interferometer) into a third recording camera. How can one verify that each imaging system is properly aligned? Can the entire imaging system be moved to a different location and easily realigned? Because this diagnostic is designed for use by many operators, its setup and use needed to be operatorindependent. Careful forethought would minimize the amount of time spent on optical alignment. As shown below, a well-written checklist would allow any optical person to guarantee the test director that he was ready for the next shot.

This diagnostic was originally designed to be a velocity interferometer to be recorded by two streak cameras at one target chamber port. Later, the design was expanded to allow for operations at other ports. Additionally, emission light could be recorded onto a third streak camera. Any of these three streak cameras could be exchanged for high-speed framing cameras to record movies of target interactions.

VISAR measures the velocity of a moving surface by recording its Doppler wavelength shift. The NIF VISAR diagnostic will be the primary diagnostic for timing the shocks induced into an ignition capsule. ${ }^{1,2}$ VISAR images will show stronger shocks overtaking weaker ones, which is the basis of the plan for setting the temporal pulse shape for the NIF drive lasers. A VISAR looking into liquid deuterium very accurately measures the speed of an approaching shock; the cold liquid is transparent, while the shocked deuterium is a highly reflective metal. Line-imaging VISAR interferometers have been fielded to measure shock velocities, shock breakout times, and emission of 1- to 5-mm targets. Figure 2 shows the locations for an imaging VISAR system used to collect images from inside a 10-m-diameter

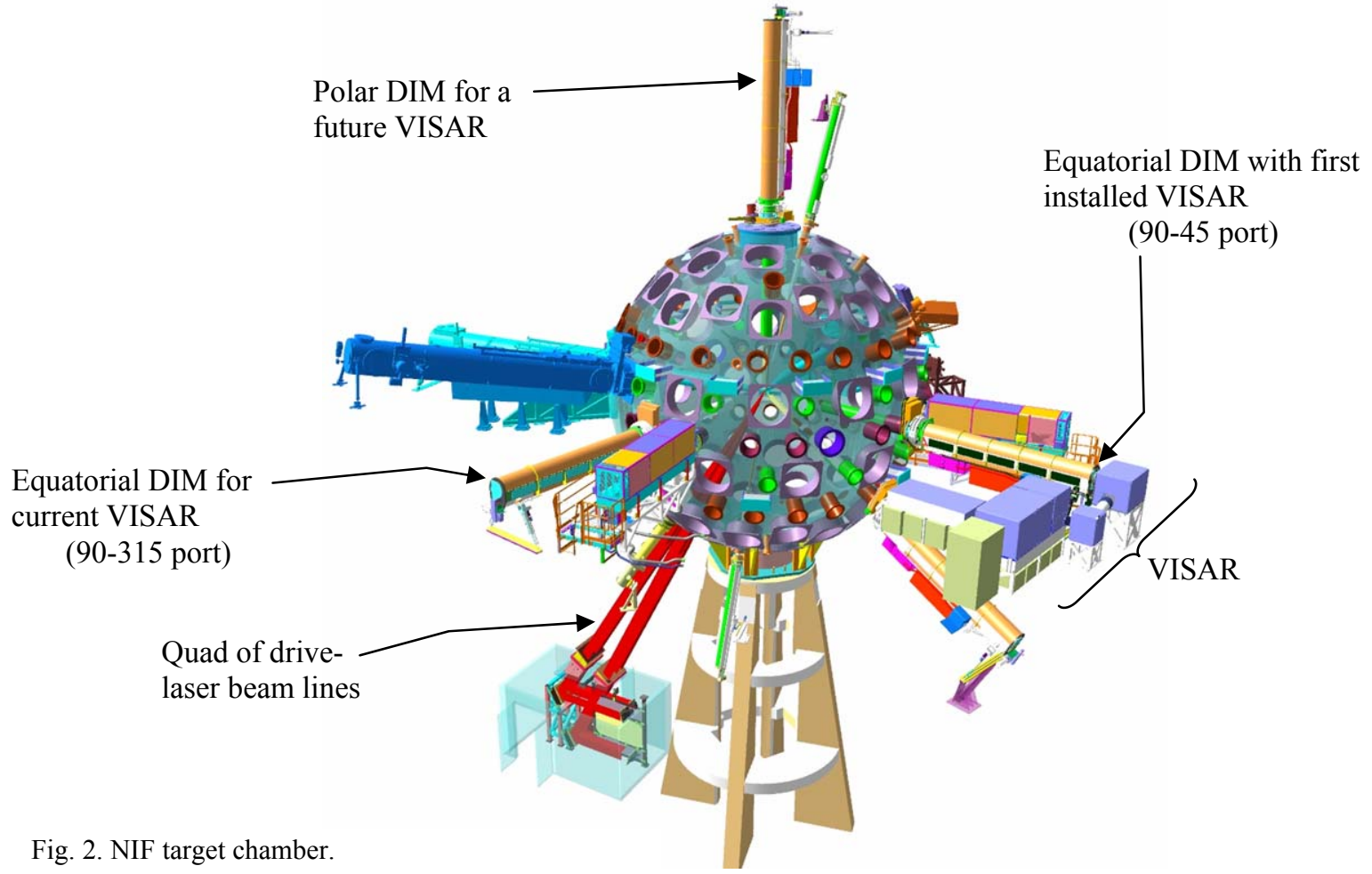


vacuum target chamber at NIF.,4,5,6,7 The first location was the 90-45 chamber port position. Four drive-laser beams entered into the NIF target chamber through one of the largest ports at the bottom of the chamber and were focused onto a sample target mid-chamber.

The Diagnostic Instrument Manipulator (DIM), located at the equator, was designed for multiple users. A two-stage VISAR DIM cart, including lenses and baffles, can be easily loaded and unloaded from the DIM like a torpedo. The DIM uses motor-controlled bipod legs for beam steering, allowing the imaging system to look anywhere within a $4 \times 4$ $\times 4$-cm volume at the target chamber center (TCC). Mirror M1 is oversized to allow for this steering; however, doublet lens L3 must be shifted $25.4 \mathrm{~cm}$ to find the correct optical axis. M1 has two tilting actuators under remote control. L3 has two remotely controlled tilt and two translation actuators. M2 is $50.8 \mathrm{~cm}$ lower in elevation than M1. M2, with two remote tilting actuators, sets the optical beam height for the interferometer table.

Figure 3 shows the ray trace layout of this imaging VISAR diagnostic that will be used at the 90-315 chamber port location. The light from the TCC is collected by a fused silica triplet lens (L1) and exits the DIM through a 5-cm-thick fused silica vacuum window. An intermediate image, formed inside the DIM, is picked up by a fused silica doublet lens (L3) placed in front of the first turning mirror (M1). A second turning mirror (M2) lowers the optical path to the interferometer table height.

The L3/M1 and M2 boxes, kinematically pinned to the floor, are detachable so that other diagnostics may use this same DIM location. The interferometer table is bolted to the floor. The probe laser table is located outside a containment wall. Optical fibers feed light from the probe laser onto the interferometer table. All enclosures for the DIM vacuum gate valve, vacuum window, L3/M1 box, M2 box, interferometer table, and probe laser table have safety interlock switches.

To minimize damage to expensive optical components, turning mirrors M1 and M2 (and turning mirrors M8 and M9 placed in front of the streak cameras) have special coatings that do not reflect the 1053-nm, 527-nm, or 351-nm NIF drive-laser wavelengths. All optical elements from TCC to the beamsplitter, BS1, on the interferometer table (including turning mirrors M1 and M2) are fused silica. This material minimizes radiation darkening caused by the intense X rays from some NIF experiments. Narrowband filters (3-nm band-pass) and cutoff filters are placed close to the dove prisms (used for image rotation), where the relayed light is almost collimated. This adds extra background light protection to the VISAR streak camera data from NIF drive lasers.

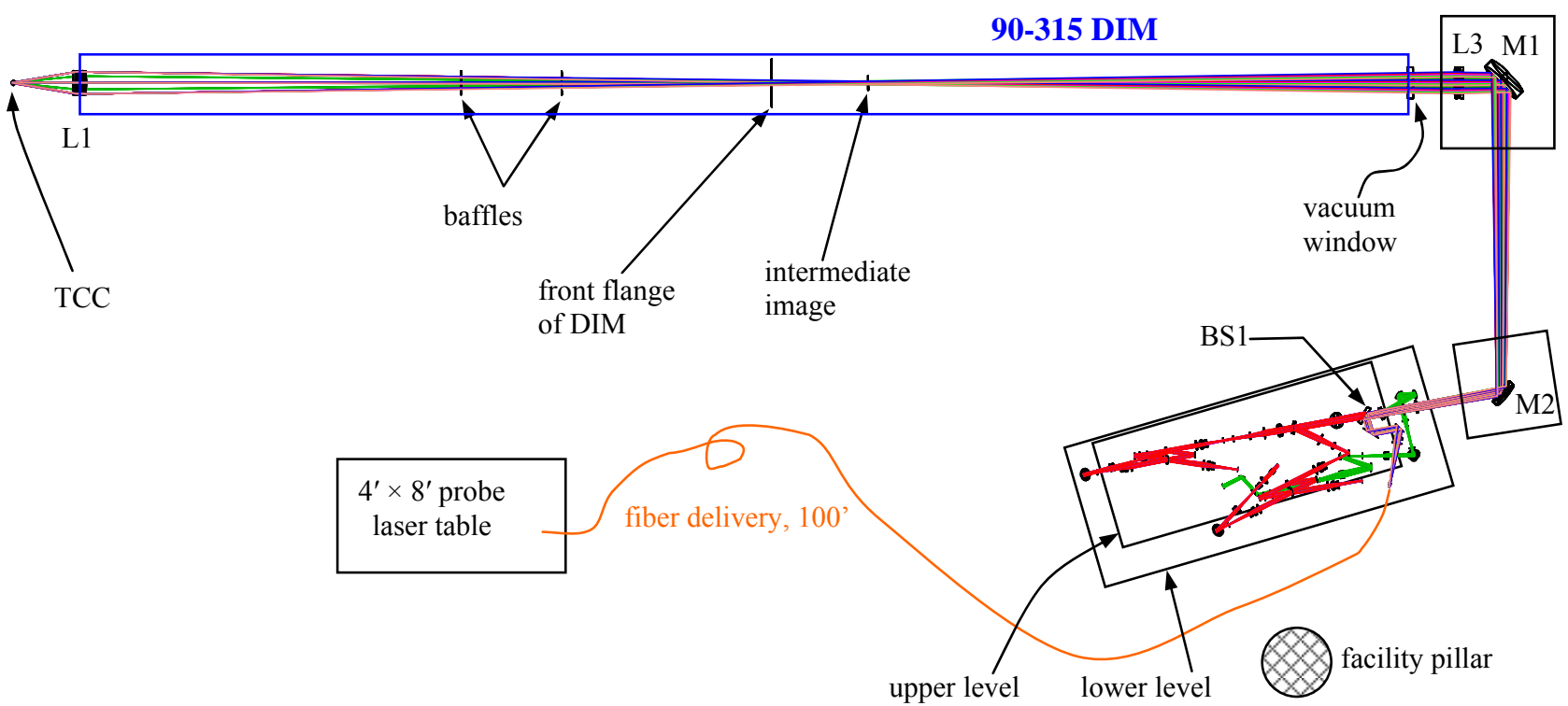

Fig. 3. VISAR optical relay system. Streak cameras are located on the upper level, interferometers on the lower level. A red diode laser, introduced in front of the three streak cameras, is sent to TCC to check alignment. The probe laser table is sited remotely. The red ray tracing is VISAR, and the green ray tracing is the thermal-imaging diagnostic. 
The special reflective broadband coatings of these turning mirrors, which reject the unwanted NIF drive-laser wavelengths, allow for other thermal-emission light produced by the target to enter onto the interferometer table. A diagnostic has been designed to collect a $105-\mathrm{nm}$ range of wavelengths at a wavelength region not contaminated by the VISAR probe laser. The range is from $540 \mathrm{~nm}$ to $645 \mathrm{~nm}$. Light is split off from the optical system using a specially coated beamsplitter (BS5, shown in Figures 1, 4, 5, 6, and 7) that transmits the VISAR light and reflects the 540- to $645-\mathrm{nm}$ band. No loss of VISAR intensity occurs. Using a band of wavelengths longer than the 659.5-nm VISAR laser would reduce signal sensitivity at the recording streak camera (S-20 extended red response). The thermal imaging collects light from a 2-mm object at $\mathrm{f} / 5$. Streak cameras perform VISAR and thermal-imaging recording.

\section{OPTICAL LAYOUT}

Due to the desire to collect light at $\mathrm{f} / 3$, which maximizes light gathered after shock breakout, and in consideration of the 500 -mm stay-out zone at the TCC, the first fused silica triplet lens (L1) is $20 \mathrm{~cm}$ in diameter. Diffraction-limited resolution (down to a few $\mu \mathrm{m}$ ) for 1- to $5-\mathrm{mm}$ objects is required at a distance of $22.9 \mathrm{~m}$. Relay lenses and turning mirrors bring this light onto an interferometer table. Two interferometers are laid out on the bottom level of the table and measure different velocity ranges. For temperature and vibration stability, interferometer optics are mounted onto kinematic plates that touch the table at only three points. Due to space constraints required by the many optical elements of the two interferometers, they are mounted on a lower level, and the recording streak cameras are mounted on a second level (see Figures 1, 4, 5, and 7). Dove prisms are located between the two optical breadboards to allow image rotation. On the lower level, beamsplitter BS2 (Figure 6) directs the light into two separate interferometers. Each

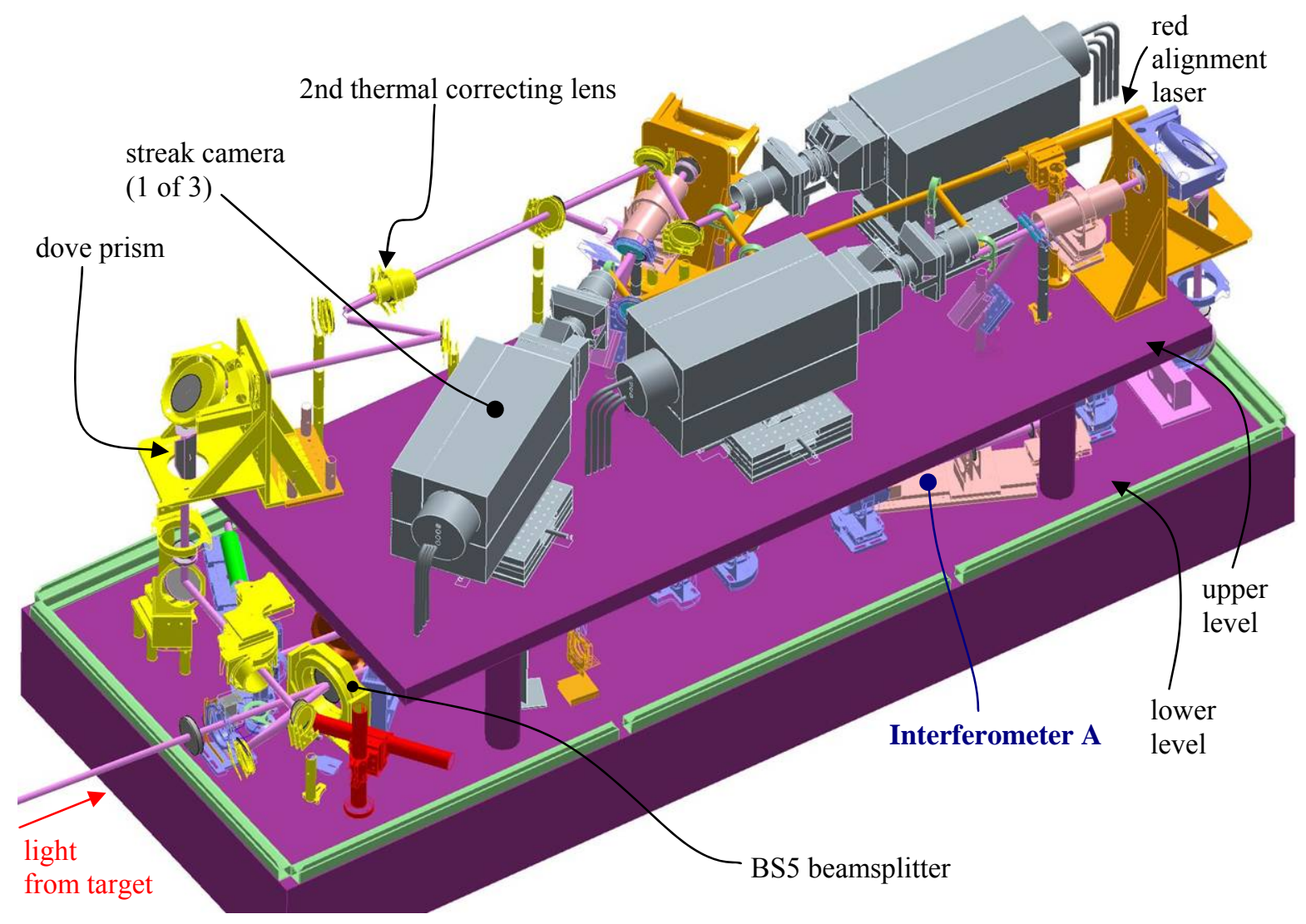

Fig. 4. Interferometer table has 2 levels. Lower level table is $4 \times 10 \mathrm{ft}$. This view is from the same perspective as that of the ray tracing in Fig. 1. 


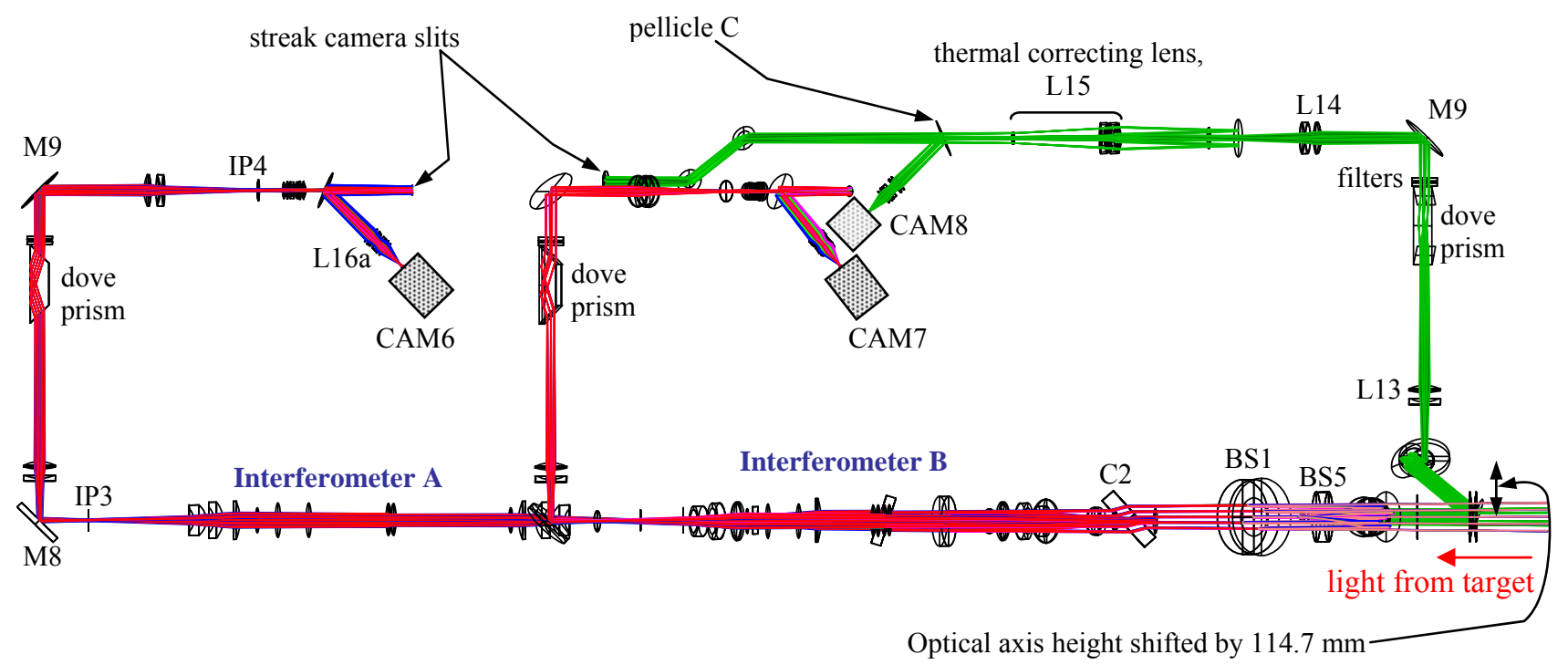

Fig. 5. Side view shows some of the many optical components inside the interferometer enclosure. Optical components are mounted onto two stacked breadboards (not shown). Red ray tracings are for VISAR. Green ray tracings are for thermal imaging. Only a few of the optical components are labeled. Filtering is done above the dove prisms, where the light is nearly collimated. Light from TCC enters from the right.

interferometer can have its own etalon (different velocity range) and image rotation, using a 37-mm aperture dove prism (see Figures 1, 4, and 5). A 1-1 optical relay is placed after the interferometer (image plane IP3) and relays the image from the lower to the upper breadboard level (image plane IP4 seen in Figures 1, 5, and 7). The dove prism is placed in the waist of the collimated section of this $1-1$ optical relay.

The interferometer uses a delay etalon in one of its legs, so that at the recombining beamsplitter, fringe shifts will move as the Doppler-shifted wavelength changes. Light is collimated at this beamsplitter. The white light source \#1 (Figure 6) is used to achieve interference fringes without the etalon inside the interferometer. Filtered white light minimizes the number of fringes present. The center of this white light fringe pattern is chosen as a reference. After centering the white light fringes at CAM2 and at CAM4 (Figure 7), the etalon is inserted in front of mirror M10 (Figures 6 and 11). M10 is then translated on a precision stage to set the back-off distance that the etalon requires. This occurs when the white light fringes reappear after retilting mirror M10.

Image plane IP2 is assigned as the primary reference plane, where a U.S. Air Force resolution pattern can be inserted. The M14 mirror is mounted onto a vertical stage and can be dropped down to prevent any light from leaving the interferometer enclosure. This M14 mirror is used to dry-run the VISAR and thermal-imaging diagnostics because access time to view an object at TCC through the DIM is very limited. Both high-power laser operations as well as lowpower laser alignment can be performed within the interferometer enclosure. When TCC access is available, focusing and steering of the L1 lens overlaps the object seen on top of the IP2 resolution pattern (see example discussed in Section 4).

Lenses L11 and L12a (Figures 6 and 11) collimate the light through the interferometers. Collimation is checked by introducing the thickest collection of etalons into only one leg of an interferometer. Good collimation is achieved when the images from the two interferometer legs have the same magnifications as seen by the monitor charge-coupled device (CCD) cameras (CAM2 and CAM4). The procedure is to vary the spacing between IP2 and L11, then apply a refocus of the final lens in front of the monitor CCD cameras. This step provides the best contrast for the fringes. 


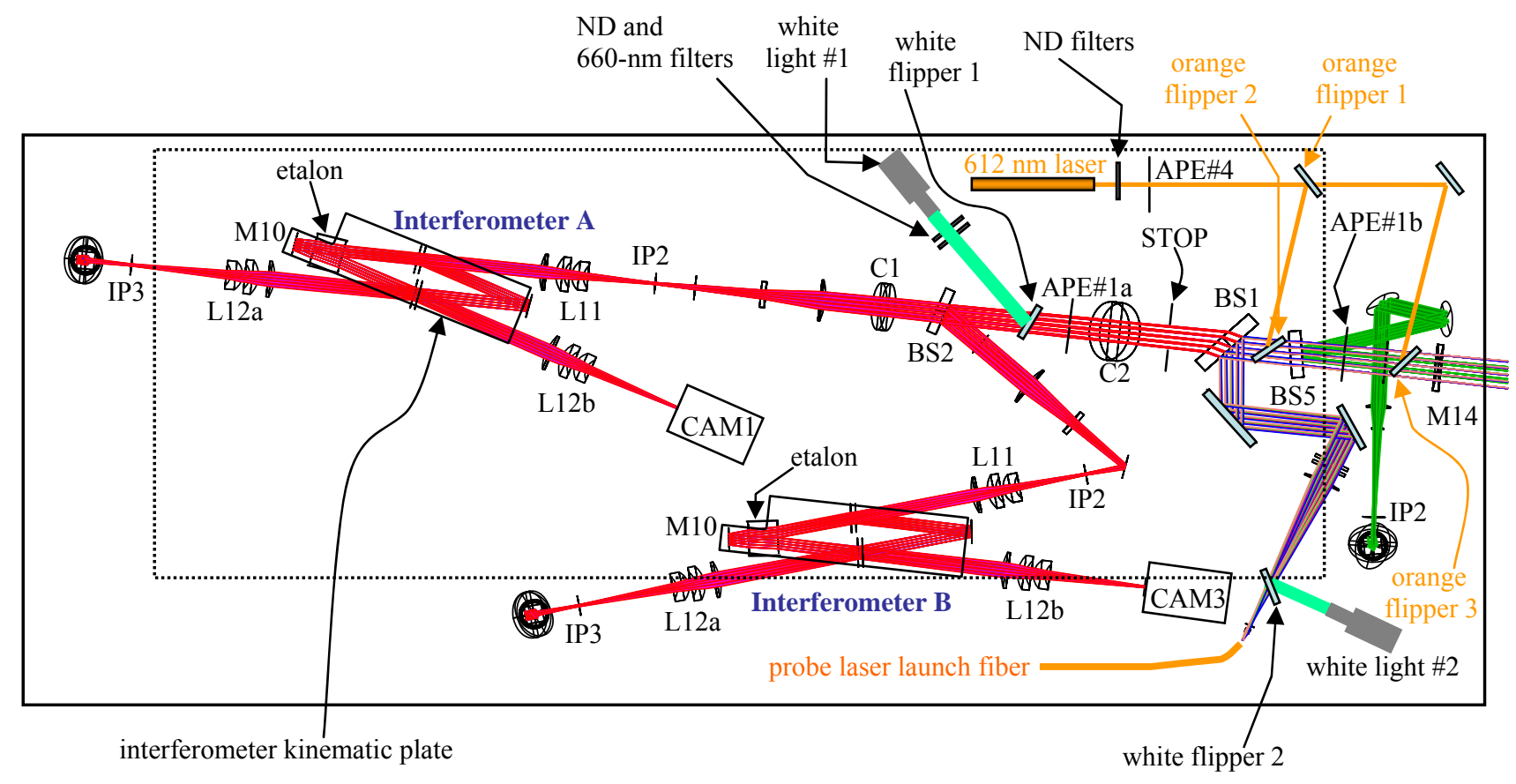

Fig. 6a. Layout of the laser alignment system on the lower level of the $4 \times 10$-foot interferometer table. Only optical ray tracing from the lower level of the interferometer table is shown. Upper level outline appears as a dotted line. The orange alignment laser is introduced close to a stop of each branch of the optical system and is sent into the slits of the streak cameras. Centering of the orange laser is checked at many apertures. White light \#1 is used to generate white light fringes for the interferometers. White light \#2 is used to illuminate the object at TCC. Because of laser speckle, fine focus of the L1 lens is easier with narrowband filtered light centered at $660 \mathrm{~nm}$ than with the 660 -nm laser diode light.

The streak cameras (Figures 4 and 7) are mounted onto rails for focus adjustment. A 50-mm macro lens, mounted to a translation stage, relays the light from the last intermediate image plane onto the streak camera slit. Translation adjustment of this 50-mm lens allows for magnification changes. Pellicles are inserted in front of the streak cameras so that CCD cameras (CAM6 and CAM7, shown in Figures 1, 5, and 7) can document the centering, size, and orientation of the target's light that scatters off the streak camera slits. Pellicles A, B, and C are used only temporarily for setup and calibration data. Other CCD cameras (e.g., CAM2 and CAM4 in Figure 7) look into flip-in mirrors for real-time monitoring of the interferometer fringe contrast.

VISAR recordings use a $60-\mathrm{kW}, 659.5-\mathrm{nm}$ probe laser, at a wavelength far removed from the NIF drive-laser beam wavelengths. This probe laser light is brought into the interferometer enclosure by optical fibers and introduced into the optical relay system at beamsplitter BS1 (Figures 6a and 6b). To maximize the light collected after shock breakout (when the shocked surface becomes curved), it is desirable to illuminate the object with f/3 light from the probe laser. ${ }^{3}$

The lens configuration for the triplet and doublet lenses used for all these optical systems was achieved through global optimization. One optical design criterion was to achieve minimum ray bending at the inside surfaces of these grouped lens elements. Minimum ray bending between these inner surfaces relaxes the tolerance requirements. All beamsplitters, including those used within the interferometers, were positioned where the light was nearly collimated. The dove prisms were located at the waist of a relay lens pair.

\section{OPTICAL ALIGNMENT ISSUES}

Maintaining stability over a very long optical path length was challenging. The shear number of optical elements made alignment complex. Alignment checks must be done on $>60$ optical components (some having seven glass elements). 


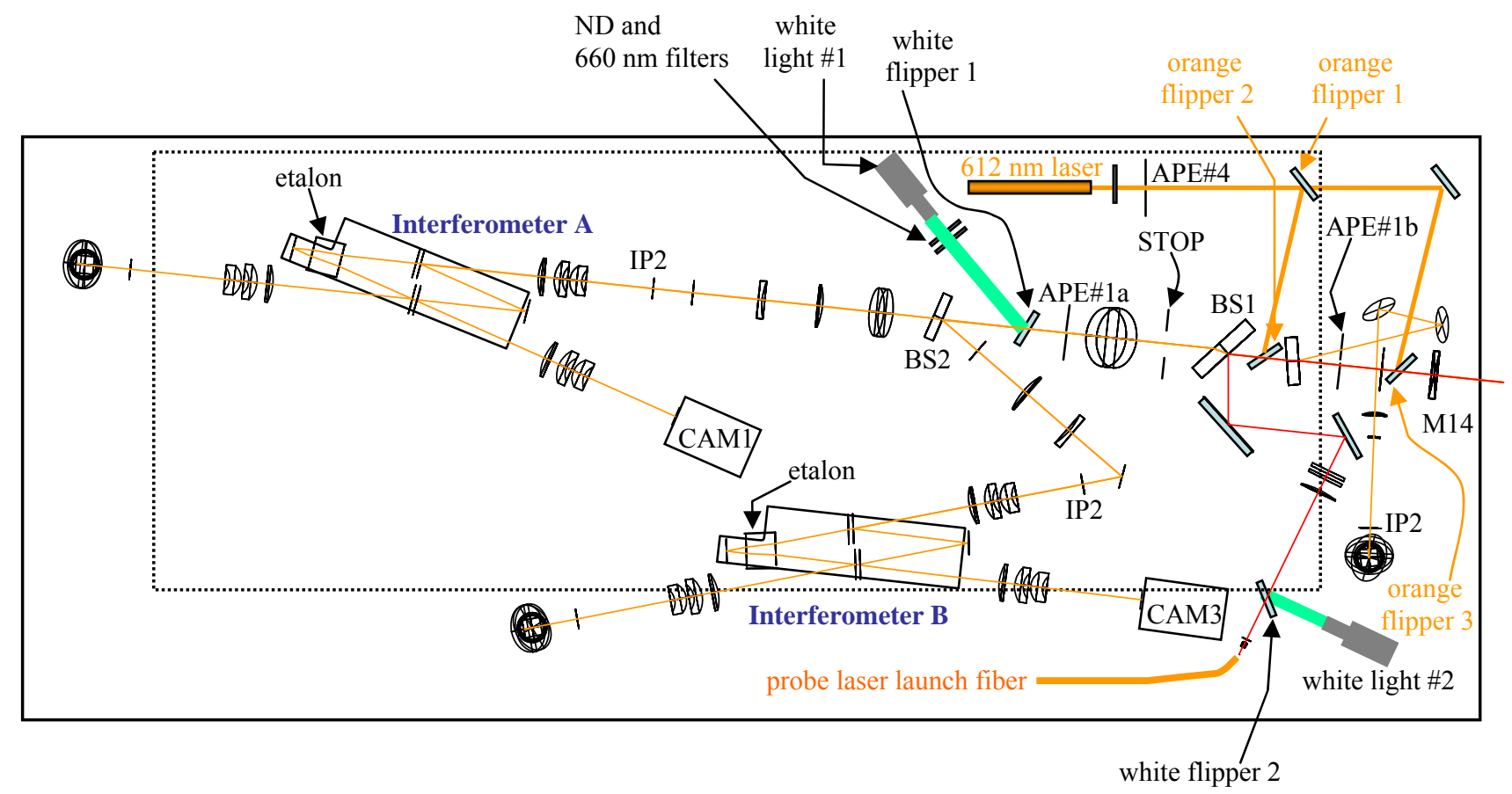

Fig. 6b. Laser alignment system, lower level. Only the orange optical axis is shown.

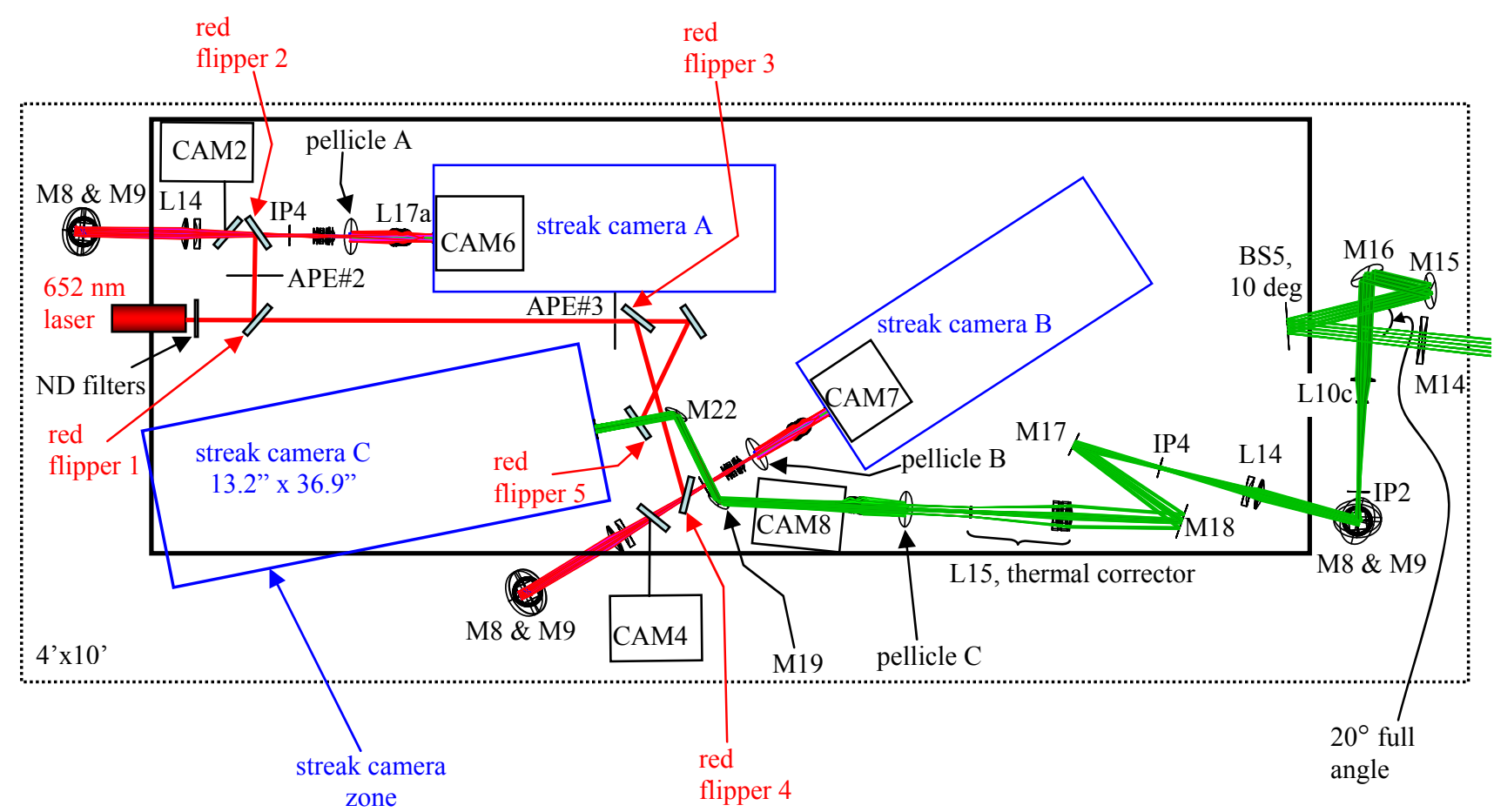

Fig. 7a. Laser alignment system, upper level. Only optical ray tracing from the upper level of the interferometer table is shown. (Some components hang over the edge of this level.) Lower level is shown as a dotted line. The 652-nm red alignment laser can be introduced in front of each streak camera via flipper mirrors and travels backward through the optical systems. Each red alignment path can be used to illuminate TCC. Laser beam centering is checked at many apertures. 


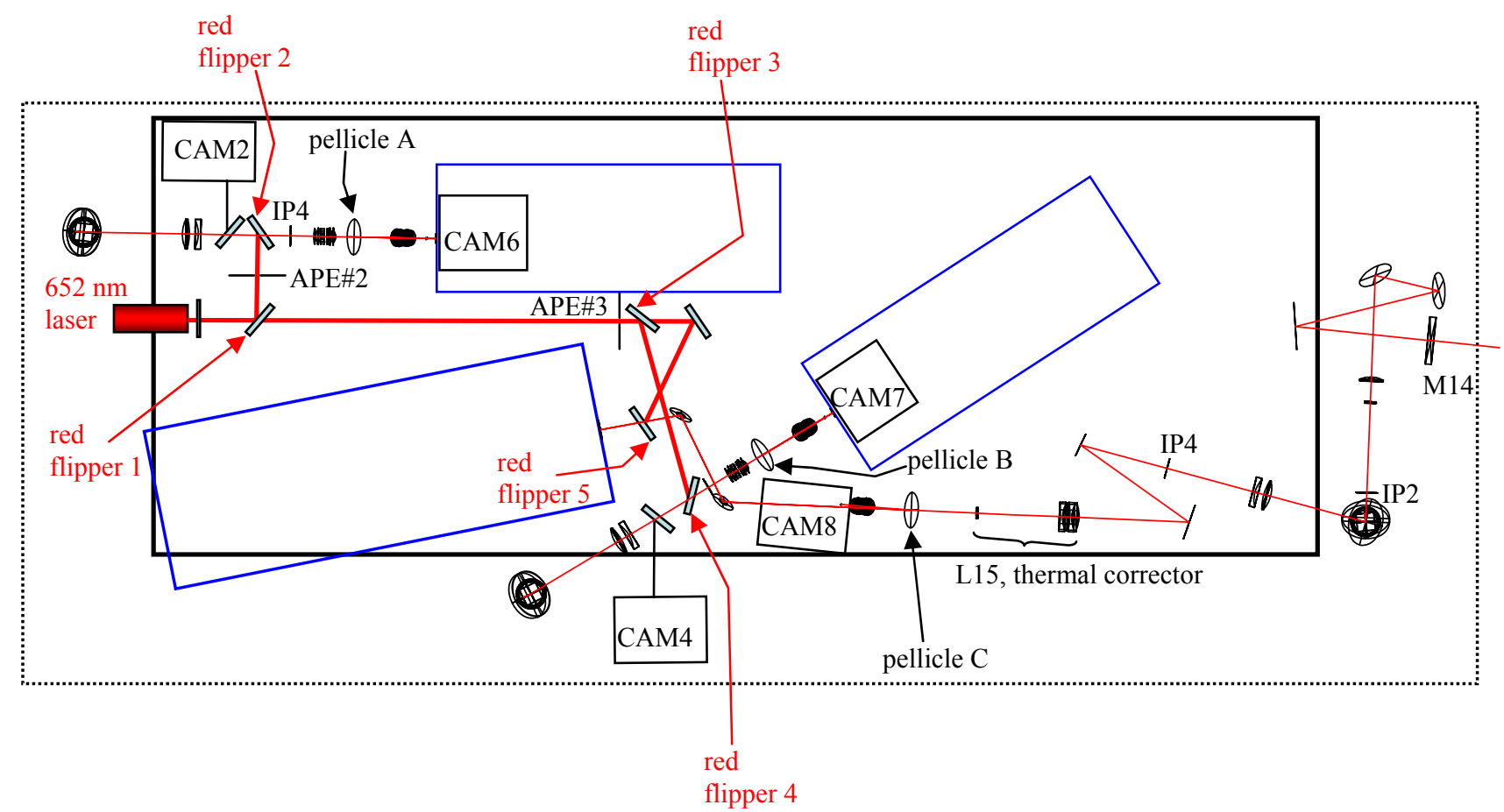

Fig. 7b. Laser alignment system, upper level. Only the red optical axis is shown.

Compound angles exist. Numerous VISAR operators are expected to use this diagnostic with different setups (etalon thickness, magnifications).

\subsection{Checking the optical axis}

Counter-propagating laser beams (orange and red) align these diagnostics to a listing of tolerances. The orange alignment laser is introduced at the entrance to the twolevel interferometer table and passes forward through the optical systems to the recording streak cameras. The red alignment laser is introduced in front of the recording streak cameras and passes in the reverse direction through all optical elements, out of the interferometer table, eventually reaching the target chamber center. Red laser wavelength is selected to be at the $50 \%$ reflection point of a special beamsplitter used to separate emission light from the Doppler-shifted interferometer light. In order to establish the optical axis, all optical lens groups are mounted on kinematic plates. After clearing all lenses from the optical axis, each lens group is individually tested for centering and tilt. By using two differently colored lasers propagating in opposite directions, very exact alignment can be achieved. The optical elements have a V-coat for $660 \mathrm{~nm}$ and provide reasonable reflections at $612-$ and $633-\mathrm{nm}$ wavelengths. Figure 8 demonstrates that an operator using only one laser beam could correct a lens decenter by tilting it. This would be insufficient alignment because a tilt can compensate a

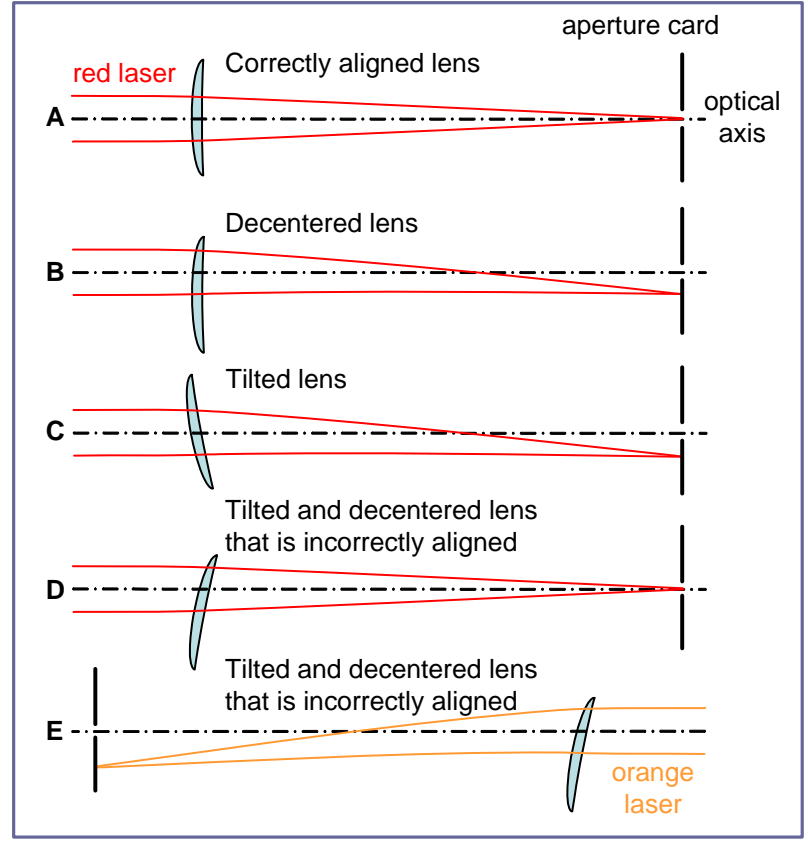

Fig. 8. How lens tilt and decenter can be used to recenter an image spot. 
decenter (Figure 8d). This would not give the correct alignment, as can be seen when using the two counterpropagating laser beams (Figure 8e).

Using a telescope or an autocollimator for alignment usually limits the viewing of alignment errors to the operator. These alignment aids would have to repeatedly be repositioned to cover the optics located on both levels inside the interferometer enclosure. Using the movable apertures to display the two-colored laser alignment errors lets multiple users probe for possible adjustments. Operators have found this method to be uncomplicated.

\subsection{Environmental problems}

Streak cameras perform both VISAR and thermal-imaging recording. When the interferometer closure is sealed, the heat generated by streak cameras causes the interferometer fringes to drift. This enclosure must be sealed when switching from alignment mode to Class IV laser operations. Thus, the interferometer sections were mounted onto kinematic plates that touched the $4^{\prime} \times 10^{\prime}$ optical table at only three points.

Optical mounting originally used $1 / 2$ " mounting posts. More stability was required, and all post-mounting structures were changed using 1" mounting posts. Earthquake restraints were added to the laser and interferometer tables, M1 and M2 boxes, and the streak cameras.

With so many optical elements, it can be difficult to determine where the misaligned elements are. Counter-propagating laser beams at two different wavelengths are used to generate reflected and transmitted spots that can be analyzed on floating apertures. Several floating apertures positioned at marked locations check the "tightness" of the cluster of twocolor laser spots. Perfect alignment is unnecessary to achieve required system resolution performances.

\subsection{Using the interferometer on different target chamber ports}

Two VISAR interferometers installed inside one enclosure have been fielded at one equatorial chamber port ${ }^{5}$ and are now being reconfigured for another equatorial port. Both have a total relay track of $21 \mathrm{~m}$. Because of the orientation relative to the DIM and facility structures, two independent layouts must be used. Figure 9 shows how the layout for the 90-45 port is accomplished. At the 90-45 port location, the interferometer table has been rotated $90^{\circ}$. Compare this layout to that of Figure 3.

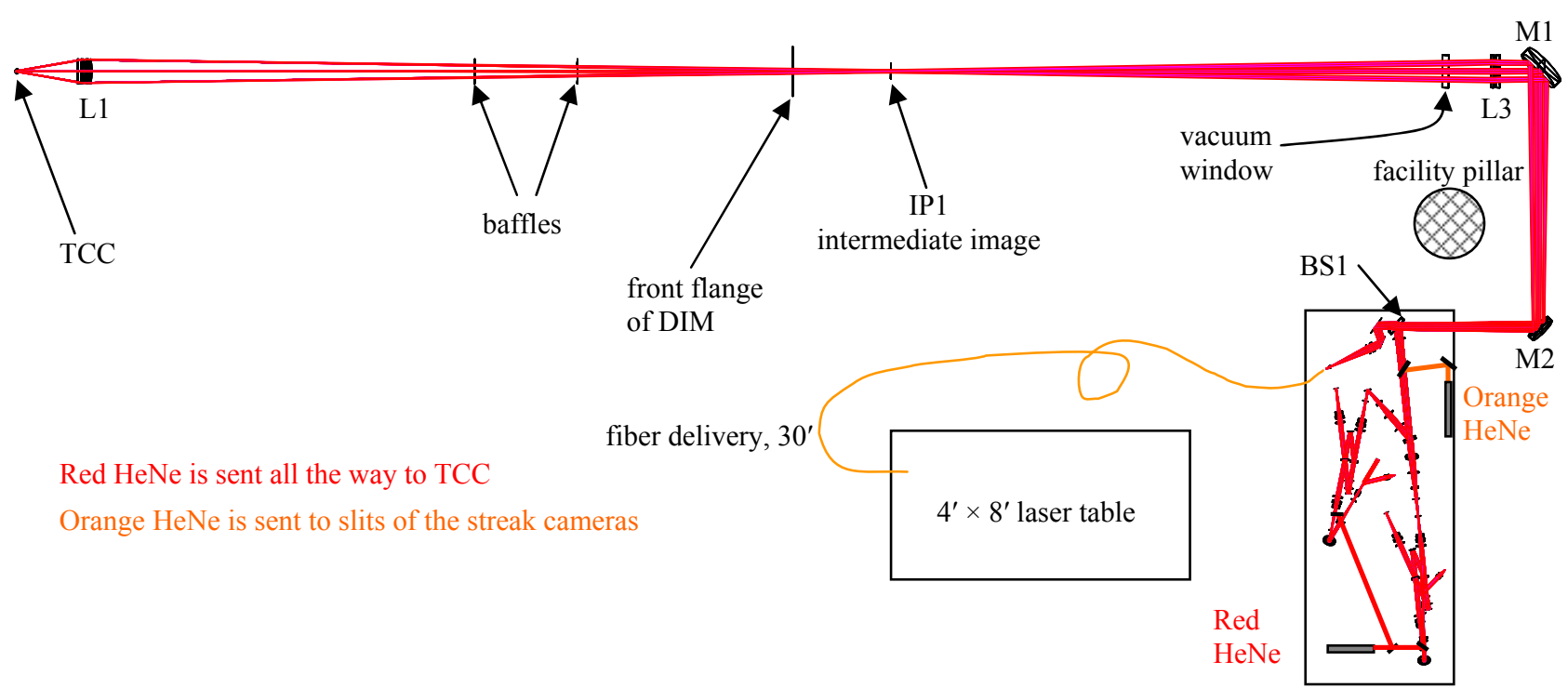

Fig. 9. VISAR optical relay system used at the 90-45 vacuum port. Thermal imaging (green system) is not shown. 
A new line-imaging VISAR relay is being designed to use a polar port with a total track of $48 \mathrm{~m}$. The same interferometer enclosure can be moved to any of the three port locations and realigned using the counter-propagating laser beam technique. ${ }^{4}$ Moving the interferometer enclosure to the polar level will require replacement of the L10 lenses. At the polar port, additional remote controls for moving relay lenses are needed. The additional optical relay lenses must be moveable within their containment boxes so that the optical axis can be checked.

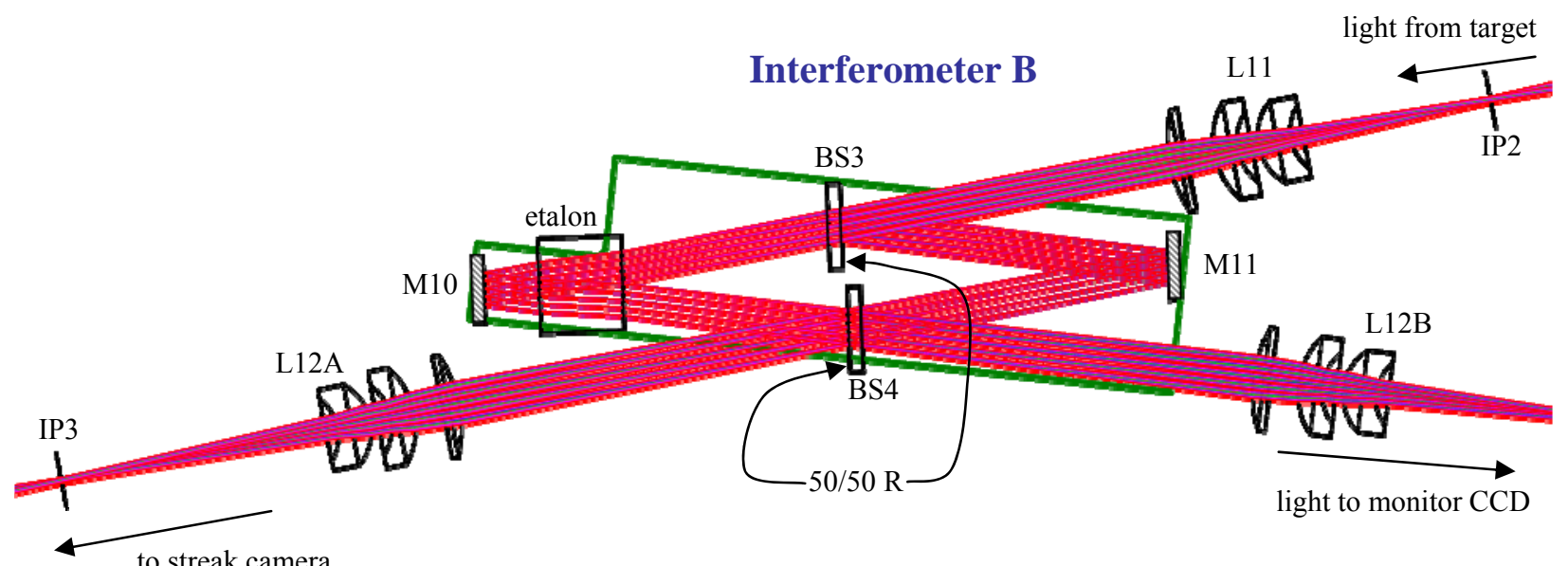

Fig. 10. Ray-tracing light through one interferometer. Outline of kinematic mounting platform appears in green.

\subsection{Alignment procedures}

Procedures have been written for different alignment topics, such as white light fringe alignment, red diode fringe alignment, dove prism alignment, aligning red diode to TCC, and alignment check points. Alignment steps are organized in a specific order. Figure 10 shows ray tracing through one interferometer. When the optical axis is being adjusted, mirror M10 is blocked and only mirror M11 and beamsplitter BS3 are used to establish the axis. Once the axis is established, mirror M11 and beamsplitter BS3 are not adjusted. To achieve the proper number of fringes across
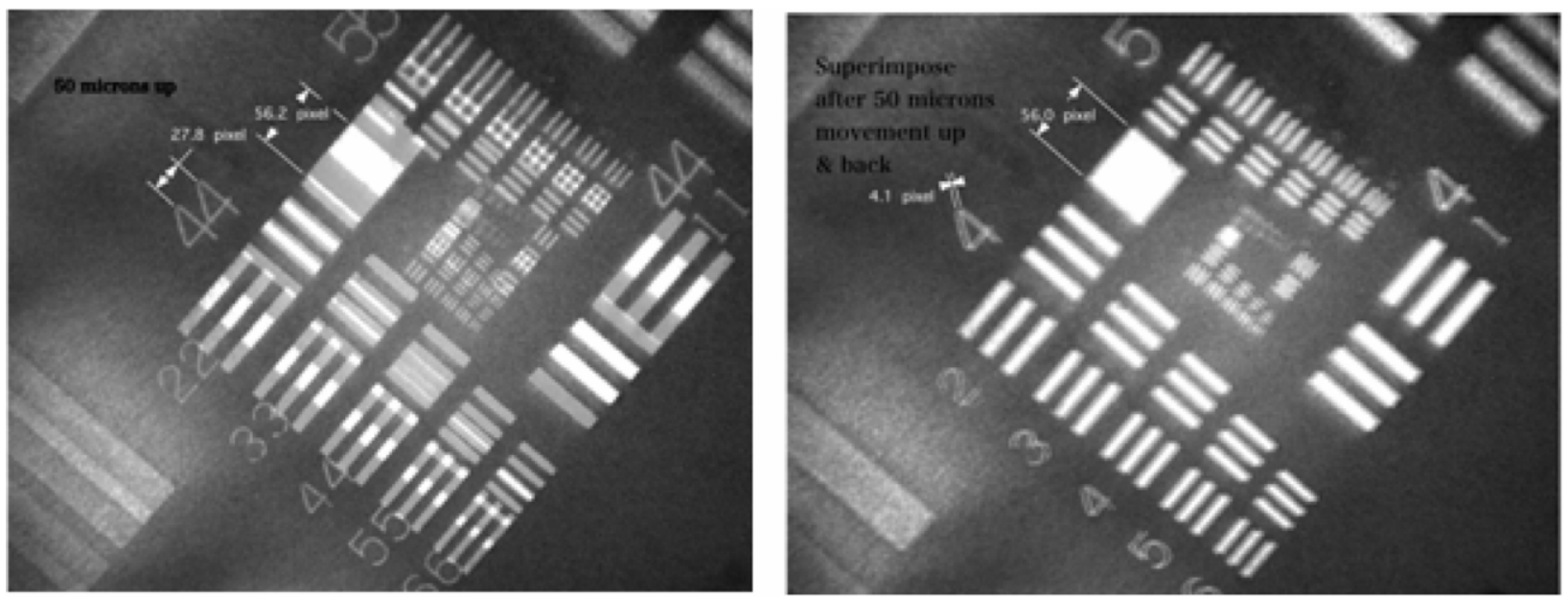

Fig. 11. White light is retro-reflected off of a U.S. Air Force resolution pattern placed at intermediate image plane IP2 and viewed with an alignment camera (placed just before the streak camera). At left, two separated images passing through different legs of an interferometer. At right, these images are overlapped. 
the image and to orient the fringes perpendicular to the streak camera slit, only mirror M10 and beamsplitter BS4 can be adjusted.

Precision alignment photos have been archived to the VISAR computer as aids in the alignment process. Archived photos of expected results from some of the alignment steps are included in checklists. Figure 11 shows an example of aligning images passing through an interferometer, as included in the topic "White Light Fringe Alignment." As different operators use the alignment procedures, updates are continually made to each alignment topic, and better notes and photos are added.

CAM6, CAM7, and CAM8 (Figure 5) are used to archive alignment targets presented to their streak camera slits. Figure 12 shows an image of a reticule placed at IP2 that is viewed by CAM7. Even though the streak camera slit is black-anodized, there is still a good reflection of IP2. This type of image proves that the imaging is properly aligned.

It is particularly important to archive the current fringe contrast and compare this photo to what has been achieved with prior setup operations. For example, different velocity ranges require different etalons. Figure 13 compares several fringe contrast measurements. The operator tries to achieve the best possible alignment within a reasonable amount of time.

Different target experiments call for the interferometers be set up to be customized: velocity ranges require a box of calibrated etalons, image rotations are controlled by the dove rotation mount, neutral density and cutoff filters are used to adjust

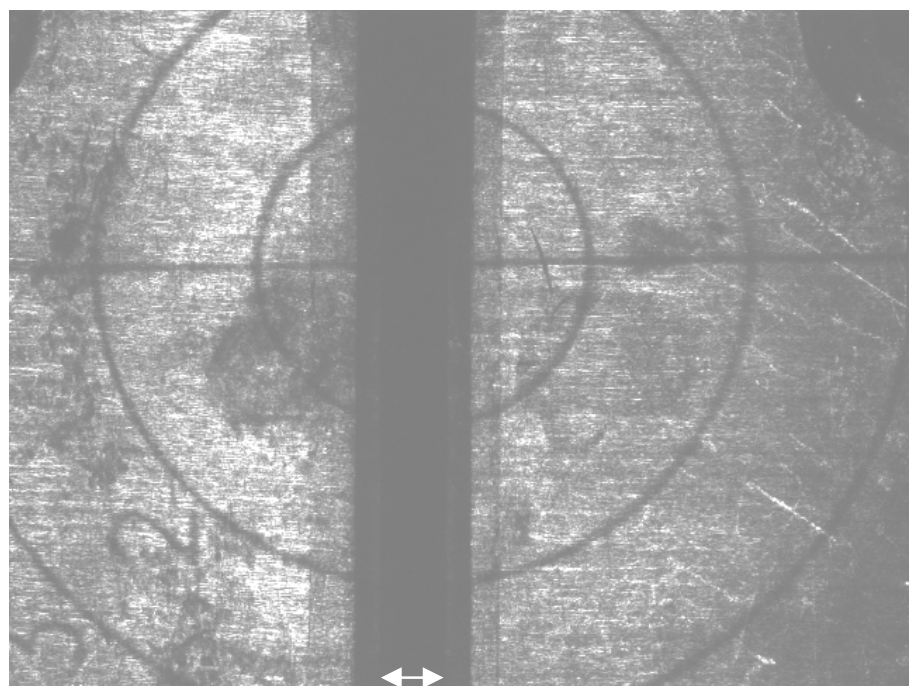
$400 \mu \mathrm{m}$

Figure 12. Resolution pattern from IP2 is superimposed on the streak camera and archived by CAM7

light levels, magnifications are determined by final focus lens and streak camera translation, etc. Each experimental setup is archived into a configuration management file for later analysis by the shot physicists. This file also includes setup images of focus and fringe contrast.
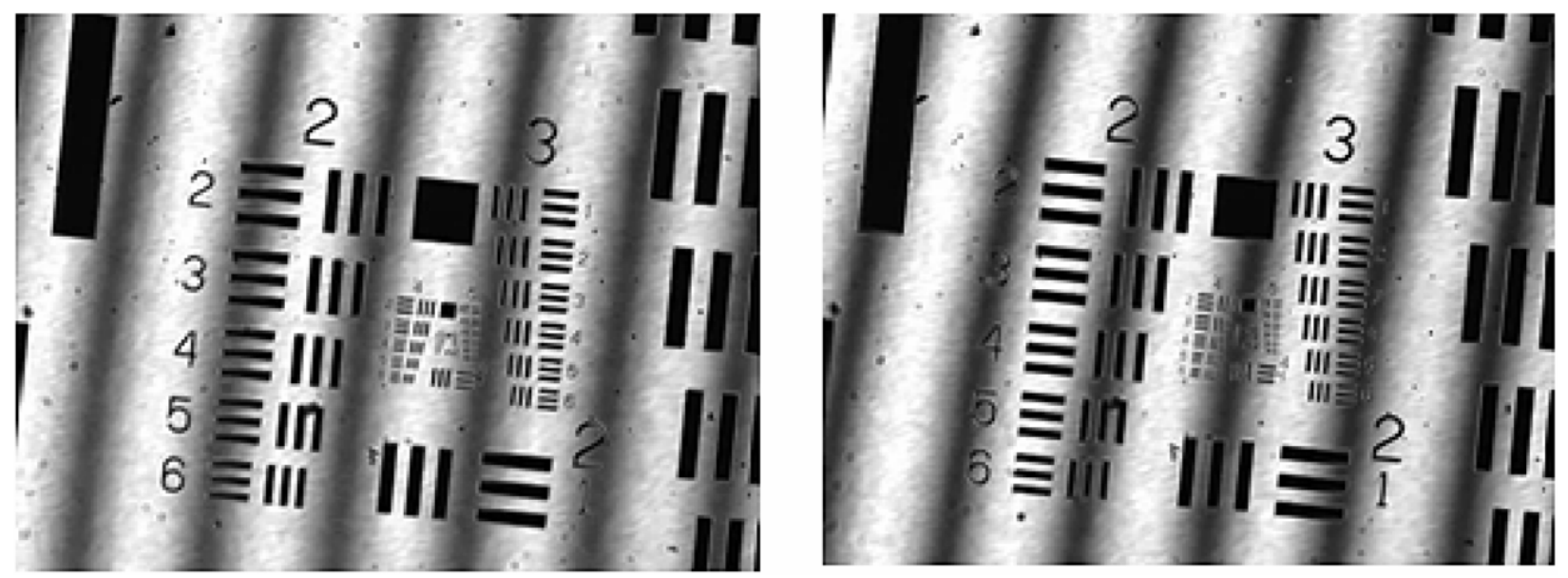

Fig. 13. Laser fringe contrast measurements show that the left image has a contrast ratio of 0.53 , whereas the right photo has a contrast ratio of 0.66 . White light fringe patterns are smoother because they contain no laser speckle. 


\section{OPTICAL ALIGNMENT RESULTS}

All optical lenses are on kinematic mounts or sliding rails, enabling pointing accuracy of the optical axis to be checked. Counter-propagating laser beams (orange and red) are used to align these diagnostics. ${ }^{4}$ The red alignment laser wavelength is selected to be at the $50 \%$ reflection point of the specially coated beamsplitter (BS5). This red alignment laser (Figure 7) is introduced at the recording streak cameras and passes through all optical elements, including this beamsplitter, on its way into the NIF target chamber. The orange alignment laser is introduced close to a field stop of each branch of the optical system and is sent into the streak camera slits. Centering of the orange and red lasers is checked at many apertures. Great care is taken to keep the orange and red laser collinear to each other. The red laser is centered on the orange laser's output mirror and vice versa.

Figure 14 shows the optical system alignment capability. The 660-nm continuous-wave (CW) laser diode (located inside the probe laser enclosure) was introduced into the optical system at beamsplitter BS1 (Figure 9). This light was sent to an Air Force resolution pattern placed at TCC. Light reflects off the bar patterns as white patterns. The reflected light provides a back light to another Air Force resolution pattern placed at the intermediate image plane IP2 (dark patterns), located just in front of the interferometer. By overlapping these two patterns, we can archive the image centering, rotation, and magnification. The monitor CCD cameras use a frame grabber in the diagnostic computer.

\section{THERMAL-IMAGING DIAGNOSTIC ALIGNMENT}

Before light enters into the two VISAR interferometers, a specially coated beamsplitter (BS5) reflects light at wavelengths from 540 to $645 \mathrm{~nm}$ into a thermal-imaging diagnostic. ${ }^{6}$ The thermal emission from the target is unpolarized light. Because a dichroic beamsplitter tilted at $45^{\circ}$

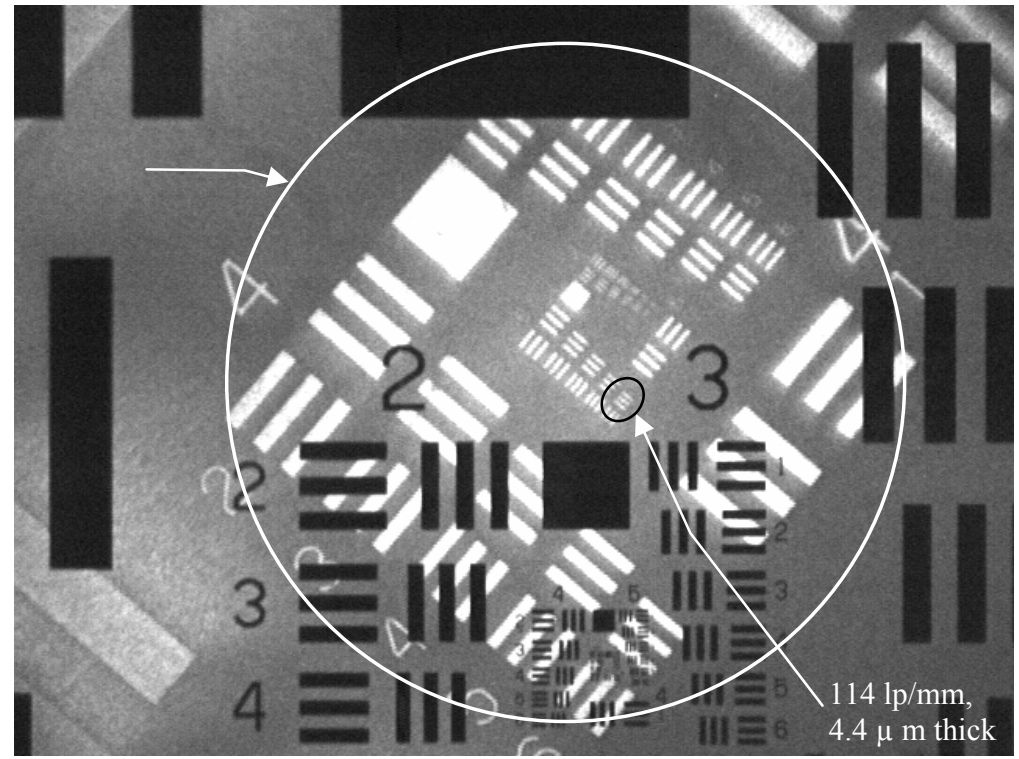

Fig. 14. Superimposed patterns viewed by CAM2 (or CAM4) with one leg of an interferometer blocked. A U.S. Air Force target placed at TCC is illuminated by a $660-\mathrm{nm} \mathrm{CW}$ laser diode (reflection mode). Reflected light from this pattern will backlight another Air Force target placed at IP2 (transmission mode). Dove prisms will provide correct image rotation onto the streak camera slits (not done for this archived image). These two Air Force target centers have been separated to show resolution differences and magnifications.

has significant wavelength separation in its reflectivity between $\mathrm{S}$ and P polarization states, we minimized the tilt angle to $10^{\circ}$.

Because fused silica optical elements are used in the first triplet relay (Figure 3), the first intermediate image planes for different wavelengths are separated by considerable distances. The L3 doublet is also fused silica, so the problem worsens before any correction can be applied. The requirement is to undo the axial color separations caused by the fused silica optical elements. Two corrector lenses (L15 and L10c shown in Figures 4, 5, and 7) on the interferometer table reunite these separated wavelength planes to provide a good thermal image. Thermal imaging collects light at $\mathrm{f} / 5$ from a $2-\mathrm{mm}$ spot on a target placed inside the target chamber. One part in 100 can be resolved over a 105-nm wavelength band.

L15 is an unusual relay lens because its object (intermediate image plane IP4) is placed at a different distance depending on its wavelength. Figure 15 shows that the distance from the lens to the streak camera slit remains constant over the wavelength range. However, the object distance changes by $57.3 \mathrm{~mm}$ over the $105-\mathrm{nm}$ wavelength range. This distance would have been larger if we did not start the color correction with the upstream L10c lens. Only the ray tracing from 


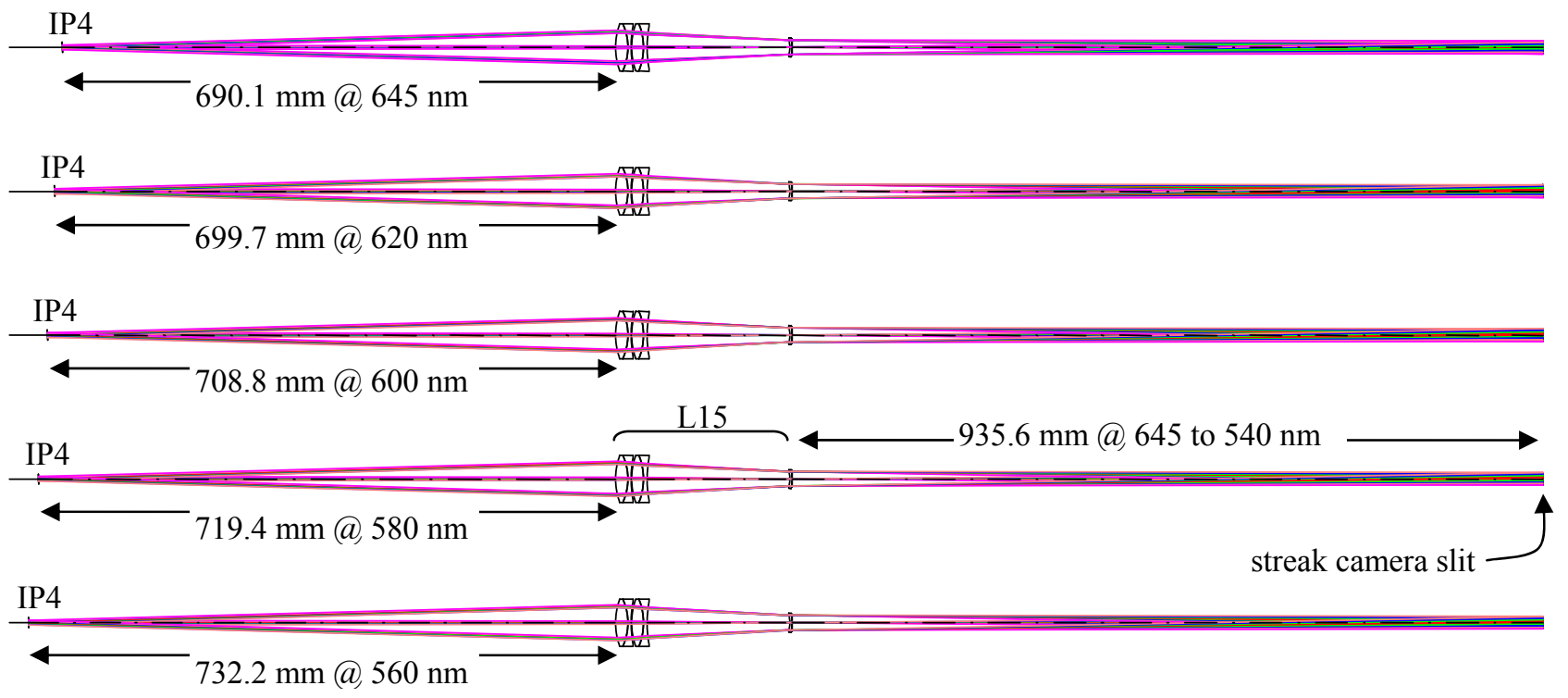

IP4

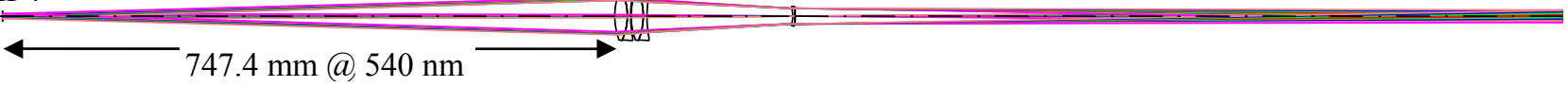

Fig. 15. Intermediate image plane IP4 (located before the thermal correcting lens, L15) is at a different location for different wavelengths. Distance from lens L15 to the streak camera slit is unchanged. This unfolded geometry does not include the four folding mirrors shown in Figures 7 and 8.

the last intermediate image plane (IP4) to the streak camera slit appears in Figure 15 (shown without four folding mirrors).

Proper focus adjustment of lens L15 can only be made using a laser wavelength, since the position of intermediate image plane IP4 depends on wavelength. Using white light with a narrowband filter would produce a blurry image. (For the VISAR diagnostic, using white light with a narrowband filter allows for optimal focus because speckle is eliminated.)

We selected a red diode laser to have a wavelength $(652.7 \mathrm{~nm})$ at the $50 \%$ reflection point of beamsplitter BS5. Its light is inserted just in front of the streak camera and travels backward through the optics on its way to the target at TCC. The orange laser is inserted (using flip mirrors) after BS5 for both VISAR and the thermal-imaging system. Its light passes through the dove prism on its way to the streak camera slits. All optical elements are on kinematic bases so that they can be easily removed to establish the correct optical axis. The advantages of a two-color laser alignment scheme are discussed in References 6 and 7.

Many flipper mirrors are used to introduce the different alignment lasers, as shown in Figures 6 and 7. The position of IP2 on the thermal-imaging system must be shifted depending on which laser provides its illumination. Inserting mirror M14 will allow some of the 652.7-nm alignment laser that has passed through one of the VISAR interferometers to reflect backward into the thermal-imaging system and illuminate a resolution pattern placed at IP2. The orange laser $(612 \mathrm{~nm})$ can also illuminate this same resolution pattern. This allows us to achieve proper focus of the L10c and L15 correcting lenses at two different wavelengths. The shift of the IP2 position at these two wavelengths is $15.7 \mathrm{~mm}$. White light \#1 is used to view white light fringes. White light \#2 sends broadband light to TCC. Monitor cameras that view the streak camera slits can archive target features. CAM8 (Figure 5) is an example of a monitor camera. Final calibration of thermal imaging will use a resolution pattern placed at TCC and illuminated by white light \#2. This illuminated target will be superimposed on top of another resolution pattern placed at IP2, as in Figure 12. 


\section{ALIGNMENT OPERATIONS SUMMARY}

The cost of not being ready for shot time is considerable. So, care must be taken to make the alignment process economical. Because of so much detail in the previous sections, we wish to summarize the most important alignment procedures in a logical sequence. TCC can be anywhere within a $10 \times 10 \times 10 \mathrm{~cm}$ cube. Both VISAR and thermal imaging can be tested independent of any access to TCC. A curved mirror (M14) placed at the interferometer enclosure entrance is designed to simulate light from TCC. The probe laser and streak cameras can be tested independently of any target chamber activities. Intermediate image plane IP2 is established as a reference surface.

\subsection{Establish optical axes.}

This is performed only inside the interferometer enclosure. Clear all lenses from the optical axes. Red and orange lasers counter-propagate and are collinear.

6.2 Test each optical group (by itself) for centering and tilt.

Perfect alignment is not necessary. Archival photos of movable apertures in preassigned locations can be used as a guide.

6.3 Insert all optical elements into the optical axes.

Research for any large clusters of alignment dots using the movable aperture at preassigned locations.

6.4 White light fringes achieved without etalons in position.

Requires resolution pattern set up at IP2.

6.5 Insert etalons and adjust fringe contrast and rotations.

6.6 Rotate image presented to streak camera slit.

Rotate dove prism and correctly align resolution pattern IP2 to streak camera slits.

6.7 Test performance of the probe laser after it has warmed up.

Take practice fringe images on streak cameras after changing delay trigger times and adding ND filtering.

6.8 Insert target at TCC.

Perform only after both the interferometers and probe laser systems have been aligned.

6.9 Perform rough alignment of target at TCC.

Adjust mirrors M1 and M2 to center red alignment laser on crosshairs on vacuum port window, doublet L3, and triplet L1 (which has permanently mounted crosshairs). Tip/tilt the DIM using bipod feet to find the target at TCC. Adjust lens L10 to overlay target features with image plane IP2.

6.10 Perform fine alignment of target at TCC.

Perform after other diagnostics and NIF drive lasers have been aligned to TCC.

6.11 Final interferometer checklist.

Recheck image focus, image rotation, fringe contrast adjustments, fringe rotations, and light filtering.

6.12 Archive alignment images to computer.

Eight CCD cameras contain alignment information.

\section{CONCLUSION}

After installing the VISAR imaging system at the 90-45 chamber port, we were able to maintain diffraction-limited optical performance. For a 5-mm field of view at TCC, 1 part in 700 was resolved at the streak camera slit. For a 1-mm field of view, 1 part in 250 was resolved. Some of the optics must work inside vacuum systems and in high-radiation environments. The flexible optical relay system allows for some air path-length changes to occur.

A thermal-imaging diagnostic using the same front-end optics as the VISAR imaging system has been designed and will be used first at the 90-315 chamber port. Correcting lenses have been developed that undo the chromatic aberrations produced by the fused silica front-end optics. A specially designed beamsplitter ensures that VISAR light is not attenuated. Using a 105-nm wavelength band, we can collect light at f/5 and resolve 1 part in 100 across a 2-mm object with our recording streak camera. No cross talk of light exists between the VISAR and the thermal-imaging diagnostics. A dove prism allows for rotating the object's image so that the streak camera slit will select the desired line profile through this image. This thermal image could also be sent to a framing camera to record a movie. The interferometer table, laser table, mirror box M1, and mirror box M2 have lifting jacks and wheels to make the entire diagnostic portable. 
All lenses can be quickly removed from the beam paths for alignment checks. The two-color laser alignment scheme is accurate and compatible with optical systems having a large number of components. Before each target event, eight CCD cameras monitor the optical performances from a remote control room.

\section{REFERENCES}

1. P. M. Celliers, G. W. Collins, L. B. Da Silva, D. M. Gold, R. C. Cauble, R. J. Wallace, M. E. Foord, and B. A. Hammel, "Shock-induced transformation of liquid deuterium into a metallic fluid," Phys. Rev. Lett. 84, 55645567 (2000).

2. D. H. Munro, P. M. Celliers, G. W. Collins, D. M. Gold, L. B. Da Silva, S. W. Haan, R. C. Cauble, B. A. Hammel, and W. W. Hsing, "Shock timing technique for the National Ignition Facility," Physics of Plasmas 8, 2245 (2001).

3. R. M. Malone, B. C. Frogget, M. I. Kaufman, P. W. Watts, P. M. Bell, J. R. Celeste, and T. L. Lee, "Design of an imaging VISAR diagnostic for the National Ignition Facility (NIF)," in Current Developments in Lens Design and Optical Engineering IV, Proc. SPIE 5173, 26-37 (2003).

4. R. M. Malone, G. A. Capelle, B. C. Frogget, R. L. Guyton, M. I. Kaufman, G. A. Lare, T. W. Tunnell, P. W. Watts, J. R. Bower, J. R. Celeste, P. M. Celliers, T. L. Lee, B. J. MacGowan, S. Montelongo, E. W. Ng, and T. L. Thomas, "Fielding of an imaging VISAR diagnostic at the National Ignition Facility (NIF)," in Current Developments in Lens Design and Optical Engineering V, Proc. SPIE 5523, 148-157 (2004).

5. R. M. Malone, J. R. Bower, D. K. Bradley, G. A. Capelle, J. R. Celeste, P. M. Celliers, G. W. Collins, M. J. Eckart, J. H. Eggert, B. C. Frogget, R. L. Guyton, D. G. Hicks, M. I. Kaufman, B. J. MacGowan, S. Montelongo, E. W. Ng, R. B. Robinson, T. W. Tunnell, P. W. Watts, and P. G. Zapata, "Imaging VISAR diagnostic for the National Ignition Facility (NIF)," in $26^{\text {th }}$ International Congress on High-Speed Photography and Photonics, Proc. SPIE 5580, 505-516 (2004).

6. R. M. Malone, J. R. Celeste, P. M. Celliers, B. C. Frogget, R. L. Guyton, M. I. Kaufman, T. L. Lee, B.J. MacGowan, E. W. Ng, I. P. Reinbachs, R. B. Robinson, L. G. Seppala, T. W. Tunnell, and P. W. Watts, "Combining a thermal-imaging diagnostic with an existing imaging VISAR diagnostic at the National Ignition Facility (NIF)," in Current Developments in Lens Design and Optical Engineering VI, Proc. SPIE 5874, 587409 (2005).

7. R. M. Malone, G. A. Capelle, J. R. Celeste, P. M. Celliers, B. C. Frogget, R. L. Guyton, M. I. Kaufman, T. L. Lee, B.J. MacGowan, E. W. Ng, I. P. Reinbachs, R. B. Robinson, L. G. Seppala, T. W. Tunnell, and P. W. Watts, "Overview of the line-imaging VISAR diagnostic at the National Ignition Facility (NIF)," in International Optical Design Conference, Proc. SPIE 6342-50 (2006).

Copyright. This manuscript has been authored by National Security Technologies, LLC, under Contract No. DE AC52-06NA25946 with the U.S. Department of Energy. The United States Government retains and the publisher, by accepting the article for publication, acknowledges that the United States Government retains a non-exclusive, paid-up, irrevocable, world-wide license to publish or reproduce the published form of this manuscript, or allow others to do so, for United States Government purposes.

This work was done in part under the auspices of the U.S. Department of Energy by the University of California, Lawrence Livermore National Laboratory, under Contract No. W-7405-Eng-48. 